\title{
Enfermedad meningocóccica: siempre presente. Cambios en los serogrupos en el Cono Sur*
}

\author{
Eduardo Luis López y Roberto Debbag
}

\section{Meningococcal disease: always present. Serogroup changes in the Southern Cone}

Meningococcal disease (MD) caused by Neisseria meningitidis is a condition with high mortality rates in childhood. Serogroup W135 N. meningitidis (MenW135) is usually associated with 1 to $8 \%$ of MD cases worldwide, and with a low carriage rate. During March 2000, an increase in the number of cases of MenW135 in Saudi Arabia was reported that coincided with the Hajj pilgrimage (Hajj-2000 strain). Mayer et al studied MenW135 strains from outbreaks related with this pilgrimage and found that all had been caused by the same hypervirulent clone (ST-11/complex ET-37). The circulation of this strain could also be documented in Latin America. In the last years, changes in serogroup prevalence have been observed in the region, the increase of MenW135 in the Southern Cone being the most significant. N. meningitidis infections of several serogroups including MenW135 may be prevented with chemoprophylaxis with antibiotics and quadrivalent vaccines. Better knowledge of the global epidemiology through the new molecular techniques, jointly with the availability of vaccines are the most relevant tools to control hyperendemic or epidemic periods of MD.

Key words: Meningococcal disease / Neisseria meningitidis / MenW135 / quadrivalent vaccines

Palabras clave: Enfermedad meningocóccica / Neisseria meningitidis / MenW135 / vacunas cuadrivalentes

\section{Introducción}

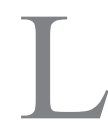

a enfermedad meningocóccica (EM), causada por Neisseria meningitidis ("meningococo"), es una de las condiciones de mayor gravedad que se observa en la niñez, con alta mortalidad. Es una enfermedad de alto impacto en la Salud Pública y una de las pocas infecciones que con baja incidencia causa tanta alarma comunitaria. En el mundo, al menos 500.000 casos ocurren anualmente con una mortalidad de $10 \%$, aunque en brotes o epidemias la tasa de mortalidad puede aumentar hasta $30 \%{ }^{1}$. Con la introducción en los calendarios de inmunizaciones de las vacunas para Haemophilus influenzae tipo b y Streptococcus pneumoniae, $N$. meningitidis en varios países se ha convertido en la causa principal de meningitis en la niñez. En los últimos años han ocurrido cambios en la epidemiología y circulación de determinados serogrupos causantes de EM como el W135, que han impactado en América Latina, especialmente en Argentina, Brasil, Chile y Uruguay. El objetivo de esta comunicación es presentar una breve reseña de la EM, los cambios en la microbiología y circulación de serogrupos, con especial énfasis en el Cono Sur.

\section{Microbiología de $N$. meningitidis con especial referencia al serogrupo W135}

N. meningitidis es un diplococo gramnegativo, aerobio, no móvil, perteneciente a la familia Neisseriaceae, que se clasifica en 12 serogrupos, según la composición antigénica de los polisacáridos capsulares: A, B, C, H, I, K, L, W135, X, Y, Z y 29E. Los serogrupos A, B, C, W135 e $\mathrm{Y}$, y recientemente el serogrupo $\mathrm{X}$, son significativos en el humano desde el punto de vista patogénico. N. meningitidis presenta pili, elemento esencial para la adherencia en el epitelio de la orofaringe. Además, esta bacteria alberga en la pared celular estructuras vesiculares que contienen la endotoxina, fundamental en la patogénesis de la enfermedad.

$N$. meningitidis puede ser clasificada en serotipos, serosubtipos e inmunotipos, según la composición antigénica proteica de la membrana externa. Actualmente los métodos moleculares genómicos disponibles, como el " $\mathrm{mul}$ tilocus sequence typing" (MLST), permiten identificar diversos complejos clonales o tipos genéticos. El MLST permite identificar los llamados linajes hiperinvasores ${ }^{2-4}$. El MLST es usado actualmente para monitorear estos linajes como parte del estudio de los complejos clonales, transformándose así en una herramienta relevante para el conocimiento de la epidemiología global de la EM. N. meningitidis serogrupo W135 (MenW135) es habitualmente asociada en el mundo con el 1 al $8 \%$ de los casos de EM esporádica y con una baja tasa de portadores. Además, el MenW135 era considerado un patógeno de bajo potencial para causar EM invasora o brotes epidémico mayores (habitualmente menos de 10 casos) ${ }^{5}$.

Durante marzo de 2000 en Arabia Saudita se informó un aumento del MenW135 coincidente con la participa-
Buenos Aires, Argentina.

Sociedad Argentina de Infectología Pediátrica y Departamento de Medicina del Hospital de Niños "Dr. Ricardo Gutiérrez" (ELL). Director Médico América Latina para la Prevención de la Enfermedad Meningocóccica y Pertussis, Sanofi Pasteur (RD).

\section{*Artículo por invitación.}

Eduardo López declara la inexistencia de conflictos de interés y de fuentes de financiación para la elaboración de este artículo. Roberto Debbag es empleado del Laboratorio Sanofi Pasteur. Los autores declaran no tener otra afiliación o compromiso financiero que involucre al tópico tratado.

Recibido: 27 de noviembre de 2012 Aceptado: 27 de noviembre de 2012

Correspondencia a: Eduardo Luis López eduardoluislopez@fibertel.com.ar 
ción en la peregrinación a la Meca, Hajj, donde concurrieron 1,7 millones de personas (1,3 millones de personas del resto del mundo). En agosto de ese año ocurrieron más de 400 casos de MenW135 entre los peregrinos que retornaron a sus países y sus contactos, en 16 países (Inglaterra, Francia, Marruecos, Dinamarca, Suecia, Noruega, E.U.A., Indonesia, Finlandia, Bélgica, Omán, Arabia Saudita, Alemania, Países Bajos, Singapur y Kuwait) produciendo pequeños brotes epidémicos y demostrando el potencial de este serogrupo en este sentido. La cepa que produjo el brote epidémico en al año 2000 durante la peregrinación a la Meca se denominó cepa Hajj-2000. Mayer y cols. estudiaron cepas MenW135 provenientes de diversos países donde habían ocurrido los brotes relacionados con la peregrinación, mediante serotipificación y marcadores genéticos ${ }^{6}$. Todos los brotes asociados fueron producidos por el mismo clon hipervirulento, el ST-11/complejo ET-37 ${ }^{7}$. En América Latina también se pudo documentar la circulación de esta cepas, específicamente a través del estudio de A. P. Lemos y cols. sobre la caracterización molecular y fenotipificación de 216 cepas de MenW135 productoras de EM invasora en Brasil ${ }^{8}$. El 73\% (157 cepas) de los aislados estudiados a través de MLST correspondieron a ST-11/complejo ET-37, el más prevalente, y confirmando la circulación en Brasil de este complejo relacionado con Hajj.

$N$. meningitidis ha demostrado alta capacidad de evadir la respuesta inmune mediante variaciones antigénicas de la cápsula y de las proteínas de la membrana externa9. Los dos elementos bacterianos fundamentales para la patogénesis de EM son la cápsula, que permite resistir la fagocitosis, y la endotoxina del meningococo, 800 veces más potente que la de los bacilos gramnegativos con altos niveles de endotoxina en sangre que causan septicemias más graves. Esta bacteria sólo infecta al hombre y es su único hospedero; no existen reservorios animales. Se transmite de persona a persona por medio de aerosolización o por contacto directo con las secreciones respiratorias o la saliva. La portación o colonización del meningococo puede ser transitoria, pero en ocasiones poco frecuentes se desarrolla una enfermedad grave sistémica, que ocurre habitualmente dentro de los primeros 14 días posteriores a la colonización, y que puede evolucionar en períodos tan cortos como de un día. Los convivientes con un caso de EM, especialmente los hogareños, presentan un riesgo estimado de contagio $\sim 800$ veces mayor que el resto de la población ${ }^{10}$.

La portación está estimada en alrededor de 5 al 10\% en los períodos interepidémicos pero en algunas poblaciones puede llegar a $40 \%$, especialmente en adolescentes, la franja etaria donde se observa la mayor tasa de portación. Los niños bajo un año de edad son los que presentan menor porcentaje de colonización, dado que la portación aumenta con la edad. Además, la portación es mayor durante brotes o epidemias. El hacinamiento, las epidemias de virosis respiratoria (influenza, en particular), el humo (tabaco, combustión de biomasa) son los factores que favorecen la diseminación y facilitan la invasión del agente causal ${ }^{11}$. También existe una relación entre las infecciones meningocóccicas y el clima, que señalan un aumento de las infecciones por $N$. meningitidis en las estaciones secas con menor humedad relativa ambiente y con mayor número de partículas de polvo ambiental, según datos del Atlas of Health and Climate publicado en noviembre 2012 por la Organización Mundial de la Salud conjuntamente con la Organización Mundial de Meteorología.

En relación al estado de portación de MenW135, estudios realizados por el Centers for Disease Control and Prevention (CDC) de Atlanta, indican que podría tener un comportamiento similar al serogrupo $\mathrm{C}$, complejo clonal ET $37^{6}$.

En América Latina durante el año 2012 se están realizando diversos estudios de portación de $N$. meningitidis, a través del Proyecto de Vigilancia y Caracterización de la Enfermedad Meningocóccica (OPS-PAHEF). En Argentina el estudio será realizado en individuos de 18 a 21 años (700 sujetos). En Brasil, J. Cassio de Moraes junto al Centro de Epidemiología del Estado de San Pablo y Campiñas y el Instituto Adolfo Lutz, realizaron un estudio de portadores en 1.231 adolescentes de entre 11 y 19 años provenientes de 76 escuelas bajo la metodología de cultivo y RPC. Datos preliminares (J. Cassio de Moraes comunicación personal) muestran una prevalencia de $8,3 \%$ de portadores de $N$. meningitidis, y $0,2 \%$ del serogrupo W135.

Otro estudio de portadores realizado en San Pablo durante brotes epidémicos de serogrupo $\mathrm{C}$ en dos refinerías de nafta en el año 2010, demostró en uno de los brotes una tasa de portación de serogrupo $\mathrm{C}$ del $6,3 \%$ y del W135 del 1,6\%, mientras que en el otro brote, la tasa de portación fue del 4,9\% para el serogrupo C y del 0,4\% para el serogrupo W135 (M. Safadi, comunicación personal).

\section{Epidemiología y vigilancia epidemiológica en América Latina}

Las infecciones por $N$. meningitidis presentan una distribución universal; sin embargo, la incidencia de serogrupos resulta diferente según se trate de países en desarrollo o desarrollados. Asimismo, factores relacionados con el hospedero determinarán la mayor incidencia de $N$. menigitidis en pacientes con déficit inmunitario, aunque del total de las infecciones meningocóccicas sólo alrededor de 5\% ocurren en pacientes con factores de riesgo de infección aumentado.

La EM se presenta en forma de casos esporádicos, en brotes de magnitud variable y en forma de endemo- 
epidemia. Históricamente, el mayor número de casos se observa en el África subsahariana, conocida como el "cinturón de la meningitis", que se extiende desde Senegal hasta Etiopía, dado que se observa una tasa de ataque de alrededor de 1.000 casos por cada 100.000 habitantes. En estos países predomina en forma notable el serogrupo A, a diferencia de los países industrializados donde hay predominio de los serogrupos B y C. Cabe mencionar que el serogrupo B provocó brotes prolongados en países como Noruega, Nueva Zelandia, Francia y en algunas áreas como en Oregón, E.U.A. En América del Sur han ocurrido brotes de EM (Argentina, 1976 y 1992) causados por meningococo $\mathrm{C}$ y $\mathrm{B}$, respectivamente. Brasil ha presentado históricamente un número importante de casos por los serogrupos B y C.

En los últimos años el serogrupo W135 ha provocado brotes significativos, como ya fue mencionado, en peregrinos musulmanes a La Meca, en Arabia Saudita y desde 2002, este serogrupo ha provocado brotes en países subsaharianos en África, pequeños brotes en Argentina y recientemente en Chile $^{12}$.

El grupo de edad con mayor riesgo es el de lactantes bajo un año de edad, seguido del grupo de entre 1 y 4 años de edad. En Europa, 45\% de los casos informados corresponde a niños entre el primer mes de vida y los 4 años de edad. En algunos países como en E.U.A., ocurre un segundo pico de enfermedad en los adolescentes y adultos jóvenes ${ }^{13}$.

En América Latina existe poca información acerca de las tasas de incidencia, formas clínicas de presentación, secuelas y distribución de serogrupos, a pesar de que la EM es de notificación obligatoria en la mayoría de los países. Esto indica que la carga de enfermedad verdadera es ampliamente subestimada en la mayoría de los países de la región. Diversos factores causan el subregistro de la EM, como por ejemplo las diferentes definiciones de casos que se utilizan para las notificaciones, las dificultades en el aislamiento de $N$. meningitidis en pacientes con antibioterapia previa, las demoras en el envío o procesamiento de las muestras o, simplemente, la carencia de métodos diagnósticos adecuados en laboratorios denominados "periféricos" en los diversos países. A todos estos factores se suman las razones propias de los sistemas nacionales de notificación, y al hecho de que en la región se dispone de un sistema de vigilancia basado únicamente en el laboratorio, lo que parcializa la información del impacto real de la EM.

En la actualidad, y desde el año 2000, los datos de EM en América Latina y el Caribe (ALC) se obtienen a través de un sistema de vigilancia de laboratorio dependiente de la Organización Panamericana de Salud (OPS) denominado SIREVA II (Sistema de Redes de Vigilancia de Agentes Bacterianos Causantes de Meningitis y Neumonías) que incluye a 20 laboratorios nacionales de referencia en los distintos países. Entre sus objetivos fundamentales se encuentra el registro de prevalencia de serogrupos, serosubtipos, resistencia antimicrobiana, identificación de linajes genéticos de $N$. meningitidis. Para el SIREVA II, el Instituto Adolfo Lutz (San Pablo, Brasil) actúa como el laboratorio regional de referencia y el Instituto de Salud Carlos III en Madrid (España), funciona como el laboratorio global de referencia.

Ibarz Pavón y cols. publicaron recientemente los resultados del SIREVA II 2006-2010 y analizaron 4.735 cepas de $N$. meningitidis de ALC. La mayoría de los aislados fueron obtenidos de LCR $(71,4 \%)$, sangre $(28 \%)$ y otros sitios estériles $(0,6 \%)$ lo que demuestra que debido a que $80 \%$ de los aislados proviene de pacientes con meningitis, la carga de enfermedad basada en estos datos del sistema de vigilancia de laboratorio es parcial y subestimada. De los aislados de la Región del Cono Sur (Argentina, Brasil, Chile, Uruguay y Paraguay) 3.081 provenían de Brasil, 591 de Argentina y 326 de Chile. La distribución de las cepas en relación a la edad de los pacientes muestra que para Brasil los pacientes bajo un año fueron $17 \%$ de los casos, mientras que para Argentina, Chile y Uruguay un tercio de los casos correspondieron a esa franja etaria. La distribución de los serogrupos estudiados en ese período no fue homogénea en los países del Cono Sur. En Brasil, $66 \%$ perteneció al serogrupo C, 26,7\% al serogrupo B, en tanto que W135 e Y fueron responsables de 5,2 y 1,9\%, respectivamente, mientras que en los otros tres países, contrariamente, $66 \%$ de los casos correspondieron al serogrupo B; 7,6\% a serogrupo C; 19,6\% al W135 y 5,8\% al serogrupo $Y$ (Figura 1$)^{14}$. Recientemente, durante el año 2011 y 2012 se informó en Argentina, Chile y Uruguay un aumento significativo del serogrupo W135.

\section{Epidemiología en países del Cono Sur}

\section{Argentina}

Se reportan alrededor de 140 casos por año de EM, que representan $25 \%$ del total de meningitis purulentas incluyendo aquellas sin aislamiento bacteriano. Históricamente, los serogrupos predominantes fueron el B y el C desde el año 1992, como lo reportan en 2007 Chiavettta y cols. del Instituto Nacional de Enfermedades InfecciosasANLIS “Dr.Carlos G. Malbrán”, en un estudio sobre la distribución en serogrupos, serotipos y serosubtipos de 2.244 aislados de $N$. meningitidis obtenidos de cuadros de meningitis y/o meningococcemias durante el período 1993-2005. Durante el período 1993-1995 prevaleció el serogrupo B (66\%), mientras que entre los años 1995 y 2001 prevaleció el serogrupo C (65\%). A partir del año 2002 se documentó un aumento de los serogrupos W135 e Y que representaban en su conjunto $15,6 \%$ del total, mientras que hasta el año 2000 no superaban el $4,7 \%{ }^{15}$. 


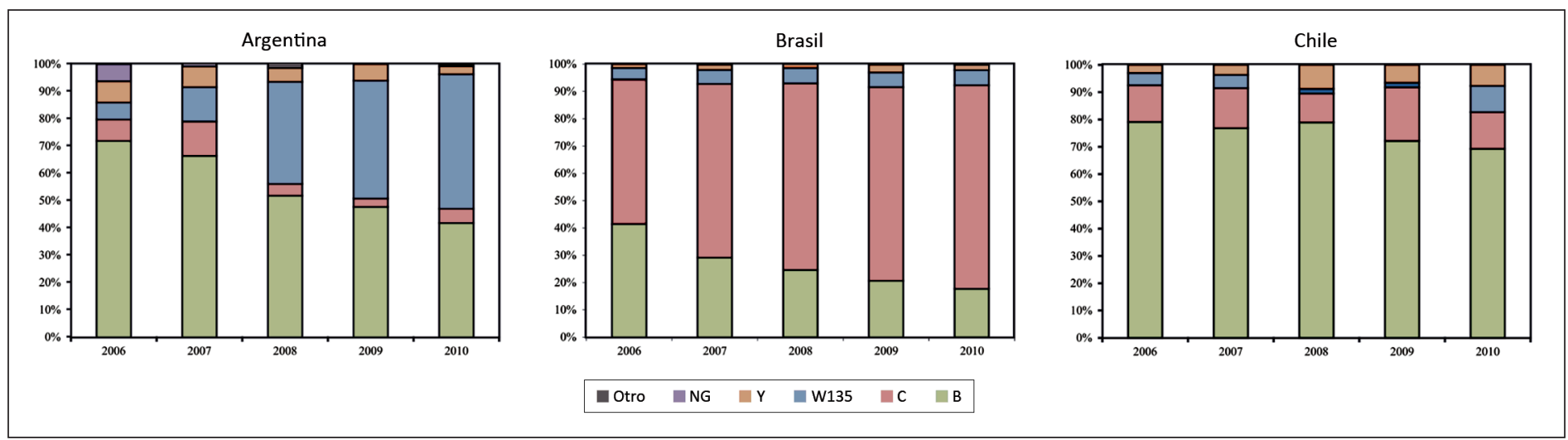

Figura 1. Distribución de serogrupos por año en Argentina, Brasil y Chile. SIREVA II: 2006 - 2010.

En el año 2009 hubo un llamado de atención por parte de Adriana Efron y Cecilia Sorhouet del Instituto Nacional de Enfermedades Infecciosas-ANLIS “Dr.Carlos G. Malbrán”, publicado en Journal of Clinical Microbiology, sobre una nueva situación epidemiológica ocurrida en Argentina desde 2006. Los autores muestran que durante 2008 se observó un drástico aumento del serogrupo W135 que llegó a alcanzar el 27,7 \% de los aislados durante ese año, y que la mayoría de las cepas provenían de la Provincia de Buenos Aires. En 60\% de los casos, la EM ocurrió en niños bajo 9 años de edad y $28 \%$ en lactantes bajo 12 meses de vida. El análisis molecular de cepas de W135 demostró pertenecer al complejo clonal ST-11 asociado a la cepa 2000 MenW135 o la llamada cepa Hajj relacionada ${ }^{16}$.

Según datos del SIREVA II publicados este año, el incremento del W135 a partir de 2006 fue significativo y más aun desde el año 2009 como se demuestra en los porcentajes de prevalencia, por ejemplo en 2006 (5,8\%), 2007 (12\%), 2008 (24\%), 2009 (41\%) y 2010 (49\%) (Figura 2).

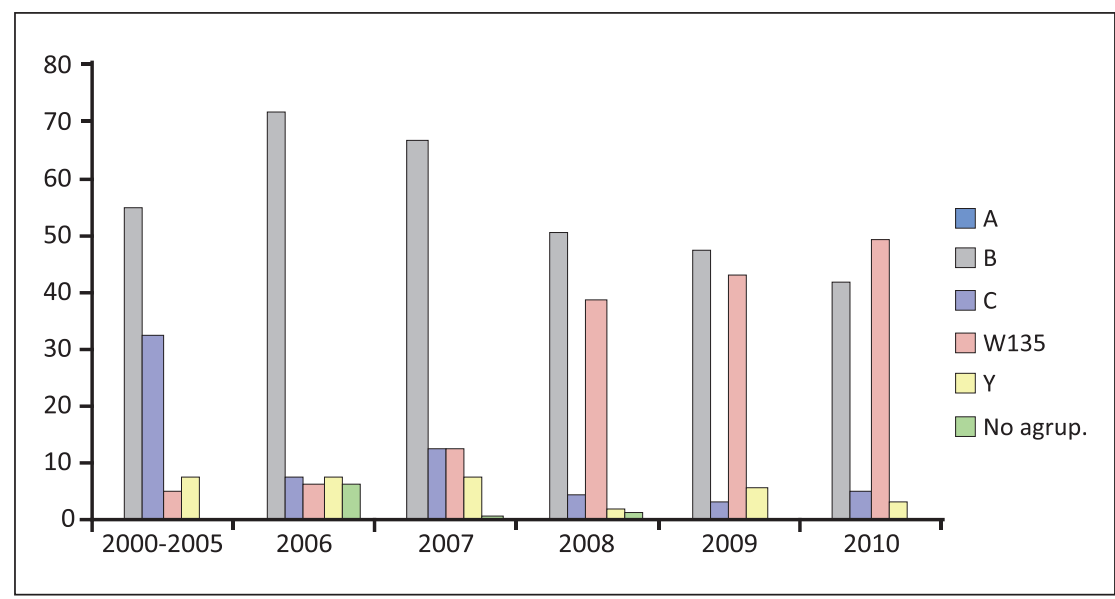

Figura 2. Distribución de serogrupos en Argentina, 2000-2010. Meningococo, estudio SIREVA.
En el año 2011 se notificaron al Ministerio de Salud Pública 153 casos de EM, con una prevalencia de W135 de $48 \%$, convirtiéndolo en el serogrupo más frecuente en ese año. Datos del Hospital de Niños Dr. Ricardo Gutierrez refieren una significativa relación del MenW135 con artritis primarias en pediatría ${ }^{17}$.

La tasa de ataque de EM es de 0,6 casos cada 100.000 habitantes por año; sin embargo en lactantes bajo un año de edad es de 13 casos cada 100.000 habitantes por año. Los niños entre 1 y 2 años representan 4,6 casos cada 100.000 habitantes por año $y$, al igual que en otros países del Cono Sur, el segundo pico de incidencia se observa en adolescentes ${ }^{14}$.

\section{Brasil}

Se considera que la endemia de la EM en América Latina es baja ( $<1$ caso por cada 100.000 habitantes), a excepción de Brasil, donde si bien el patrón epidemiológico es endémico, algunos autores lo consideran como un país hiperendémico (cercano a 2 casos por cada 100.000 habitantes) debido a la presencia de brotes epidémicos a lo largo del país. La historia epidémica de Brasil puede dividirse en dos períodos epidémicos, el primero en la década del ' 70 donde ocurrieron dos ondas epidémicas, una producida por el serogrupo C (1971) y la otra por serogrupo A (1974), en la cual se alcanzaron cifras de incidencia de 180 casos por cada 100.000 habitantes. La segunda década epidémica fue la de los ' 90 , causada por el serogrupo B, que alcanzó cifras de más de 7.000 casos durante el año $1996^{18}$.

En los últimos años la prevalencia cambió hacia el serogrupo C, manifestándose como casos esporádicos y brotes epidémicos. Según el Informe SIREVA II 20062010, el serogrupo C fue el más frecuente; en el año 2010 llegó a ser responsable de $74 \%$ del total de las infecciones notificadas con aislamientos. En los últimos años se observa un aumento en la incidencia del MenW135, según los datos de prevalencia durante el año 2002, cuando fue 
responsable de $1,5 \%$ de los aislados versus $5,5 \%$ en el año 2008 .

Este incremento se demuestra además por el aumento de la infección por MenW135 en el estado de Río Grande do Sul, donde en el período 1995-2002 fue de $3,2 \%$ comparado con el período 2003-2005, donde fue aislado de $17,8 \%$ de las muestras. Estudios posteriores de caracterización molecular de las cepas demostraron que se trataba del mismo complejo (ST-11), relacionado con Hajj ${ }^{19,20}$.

Otro ejemplo es el del estado de Río de Janeiro donde en el año 2004-2005 MenW135 era el responsable de $11 \%$ de los casos, en tanto que en el período 1988-2002 era sólo de $0,3 \%{ }^{21}$.

En un reciente estudio sobre la experiencia en Brasil con MenW135 en un período de 11 años y sobre 13.635 cepas confirmadas en el laboratorio, se aislaron 559 MenW135, lo cual correspondió al 5,8\% del total de aislados. La tasa de mortalidad fue alta y varió entre 13 y $32,5 \%$, dependiendo de la edad y la presentación clínica; $35,2 \%$ de los casos correspondió a niños bajo 5 años. De los MenW135 aislados, 14\% tenía sensibilidad reducida a penicilina ${ }^{22}$. Durante el último año la tasa de MenW135 se mantuvo estable, siendo responsable de $5,6 \%$ de los aislados ${ }^{23}$.

\section{Chile}

Al igual que en otros países del Cono Sur, Chile mantuvo, durante la década del '90, una incidencia alta de EM que varió de 2,3 casos por 100.000 habitantes por año en 1990 a 3,7 casos en el año 1996, con una mortalidad de 11,2 y $6 \%$ en 1990 y 1996, respectivamente. El serogrupo B fue el más frecuentemente aislado ( $80 \%$ de los casos), al igual que en otros países de la región, afectando en más de $60 \%$ de los casos a niños bajo 5 años de edad en esos mismos años ${ }^{24}$.

Luego, desde 2001, la incidencia de EM se mantuvo baja y estable, con notificación de 0,6 a 0,7 casos por cada 100.000 habitantes en el año 2006 y 2007 , respectivamente, con persistencia del serogrupo B como el principal.

Durante el período 2006 a 2010 según datos del SIREVA II el número de cepas aisladas de EM provenientes de Chile se mantuvo estable (entre 60 y 80 casos por año) afectando en $38 \%$ de los casos a lactantes bajo un año de vida.

En la Figura 3 se muestra el comienzo del aumento del MenW135 a partir del año 2011 donde se aislaron 19 cepas, en comparación con el año 2010 donde sólo se documentaron cinco. Durante 2012 se está observando un incremento aún mayor: en la semana epidemiológica número 46 se notificaron 48 casos de MenW135. Durante 2012, MenW145 afecta principalmente a niños bajo 5 años (52\% de los casos y $20 \%$ bajo 9 meses). Se informaron casos en seis de las 15 regiones del país. La mortalidad

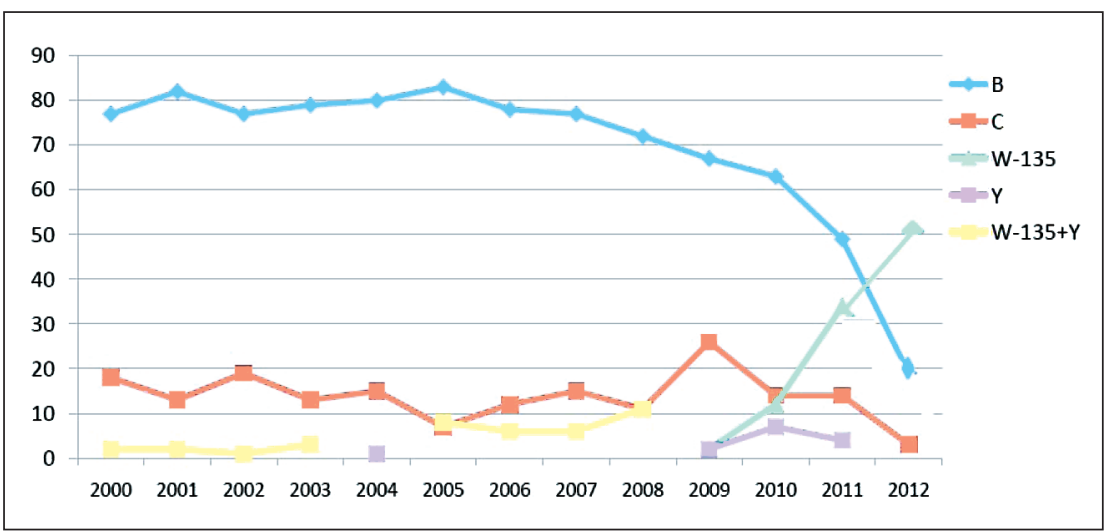

Figura 3. Distribución de serogrupos en Chile, 2000-2012.

durante este año es mayor comparada con años anteriores (21\%). La mayoría de los decesos (70\%) ocurrieron en la Región Metropolitana ${ }^{12}$.

Estudios moleculares realizados por el Instituto de Salud de Chile demostraron que las cepas de MenW135 pertenecen al complejo clonal ST-11, con el mismo linaje hipervirulento de las cepas MenW135 que circulan en los últimos años en Argentina y Brasil ${ }^{25}$ y relacionadas con las cepas Hajj ${ }^{8,16,24,25}$.

\section{Discusión}

Neisseria meningitidis es una de las causas más frecuentes de meningitis en la actualidad, además de producir formas fulminantes de meningococcemias y ser ésta una de las infecciones con mayor mortalidad en niños. MenW135 se asocia a casos esporádicos o epidemias en el mundo, si bien luego de los brotes epidémicos vinculados con la peregrinación a la Meca, se publicó una larga serie reportes de brotes causados por un complejo clonal ST-11/ET37.

Hay datos de aislamiento de estas cepas desde antes del año 2000, como lo demuestra el aislamiento en un paciente originario de Indonesia, retornado de la peregrinación en el año $1996^{6}$. Un probable "switch" capsular del serogrupo C al W135 ocurrió posiblemente desde la década del 70. Por esto, la cepa MenW135 vinculada a Hajj se interpreta que no es la emergencia de un nuevo clon sino la expansión de un complejo clonal existente con la consiguiente transmisión local. Si bien no son claros los datos relacionados con la probabilidad de que esta cepa pueda causar mayor incidencia de formas más graves como por ejemplo meningococcemia, datos aislados de diversos investigadores sugieren que estos linajes hipervirulentos estarían potencialmente asociados a mayor mortalidad. En relación a las tasas de portación del MenW135 complejo clonal ET-37/ST-11, diversos 
estudios muestran una tasa de prevalencia variable, con datos de tasas de portación de 2 a $5 \%$ como por ejemplo en el estudio de Gambia ${ }^{26}$, pero seguramente el porcentaje de portación dependerá del grado de circulación en una comunidad, ya que durante los brotes la tasa de colonización nasofaríngea es mayor. Conocer las tasas de portación y los grupos etarios más colonizados es uno de los pilares fundamentales para generar estrategias de control a través de vacunas. Experiencias internacionales europeas demuestran un control más efectivo cuando se combinan estrategias de vacunación en los grupos más afectados, como por ejemplo los niños bajo 5 años de edad y los adolescentes, quienes presentan las tasas de mayor portación y son fuente indispensable de circulación de N. meningitidis.

Para la prevención de las infecciones por N. meningitidis de los diversos serogrupos incluyendo el W135, se dispone de la quimioprofilaxis a través del uso de antimicrobianos y la utilización de vacunas.

La profilaxis antibimicrobiana ha demostrado ser efectiva en la decolonización nasofaríngea de $N$. meningitidis, razón por la cual su empleo en contactos cercanos colonizados disminuye la transmisión y la aparición de casos secundarios. Se recomienda quimioprofilaxis en los contactos estrechos del caso índice: contactos familiares, en especial niños bajo 2 años de edad, contactos de guardería o pre-escolares, en los casos de exposición directa a secreciones del caso índice (besos, compartir elementos que van a la boca), como en situaciones de resucitación boca a boca, contacto no protegido durante intubación endotraqueal, contactos que duermen frecuentemente en el mismo dormitorio que el paciente índice, y en todos los casos de contactos durante los 7 días previos al comienzo de la enfermedad.

Otra indicación es para pasajeros sentados directamente junto al caso índice durante un viaje en avión que dura más de 8 horas $^{27}$. No se recomienda profilaxis a los contactos casuales sin historia de exposición directa a las secreciones orales del caso índice (trabajo o escuela) ni a los contactos indirectos (contacto con un conviviente de alto riesgo, dado que no hay contacto directo con el paciente índice). Tampoco se recomienda a los profesionales de la salud salvo que hubiera exposición directa a las secreciones orales del paciente. Los fármacos recomendados para la quimioprofilaxis son rifampicina, ceftriaxona y ciprofloxacina, aunque esta última dependerá de la resistencia existente en esa población.

La prevención de la EM a través de diversas vacunas se ha desarrollado desde hace más de 40 años y se las considera la mejor herramienta para el control de la enfermedad. Debido a los cambios epidemiológicos globales en relación a los serogrupos productores de EM, como por ejemplo el incremento del W135 e Y, se han desarrollado vacunas conjugadas tetravalentes o también denominadas cuadrivalentes. Las primeras vacunas de la década del '70 fueron las vacunas de polisacáridos tetravalentes $(\mathrm{A}, \mathrm{C}$, W135 e Y) demostrando luego su efectividad y seguridad para evitar casos esporádicos como para el control de brotes epidémicos en niños mayores y adultos. Sin embargo, debido a la pobre respuesta de anticuerpos en lactantes, la imposibilidad de reducir o impedir la colonización nasofaríngea y por generar reacciones de hiporespuesta de anticuerpos luego de dosis repetidas, estas vacunas sólo fueron utilizadas para la prevención de infecciones en pacientes sobre 2 años de edad con factores de riesgo ${ }^{28}$. Luego de las experiencias con las vacunas de polisacáridos conjugadas con proteínas transportadoras para Haemophilus influenzae del tipo b y Streptococcus pneumoniae en lactantes, se desarrolló en E.U.A. la primera vacuna antimenigocóccica cuadrivalente conjugada con toxoide diftérico (A, C, W135 e Y-TD) que comenzó a utilizarse en el año 2005 (Menactra ${ }^{\circledR}$, SanofiPasteur) y luego en más de 30 países, mostró una evidente reducción de la carga de EM tras su introducción en el esquema de vacunación en adolescentes ${ }^{29}$. Esta vacuna está actualmente aprobada para el uso en niños a partir de los 9 meses de vida con esquema de dos dosis e intervalo de 3 meses. La segunda vacuna cuadrivalente conjugada con proteína mutante de toxoide diftérico (A, C, W 135 e Y-CRM, Menveo ${ }^{\circledR}$, Novartis) se licenció en el año 2010 para su uso a partir de los 2 años de vida con esquema de una sola dosis. Recientemente, a principios del 2012 en Europa se aprobó el uso de otra vacuna cuadrivalente conjugada con toxoide tetánico (A, C, W135 e Y-TT, GlaxoSmithKline) para uso a partir del año de vida. Las vacunas cuadrivalentes son actualmente utilizadas para la prevención de las infecciones producidas por $N$. meningitidis serogrupos A, C, W135 e Y en: pacientes con riesgo aumentado de infección; programas nacionales de inmunizaciones (por ejemplo, en adolescentes en E.U.A. a los 11 años desde 2007; esquema de vacunación universal de niños a partir de los 9 meses de edad en Arabia Saudita desde 2012) 29 $^{29}$ para prevención de infecciones en el viajero a áreas epidémicas, como por ejemplo, el cinturón de la meningitis o como requerimiento migratorio de visa para el ingreso a participar en la peregrinación a la Meca en Arabia Saudita $^{30}$. La Organización Mundial de la Salud también recomienda las vacunas conjugadas para ser utilizadas en países con tasas de hiperendemia o epidémicas ${ }^{31}$.

En conclusión, cuando ocurren casos esporádicos, la EM es potencialmente devastadora por su alta mortalidad y sus potenciales secuelas; un estudio de H. Paganini y cols. documentó la existencia de secuelas en 12\% de 126 niños con $\mathrm{EM}^{32}$. Cuando produce brotes epidémicos, es un problema de Salud Pública que no sólo causa impacto en los marcadores de la salud sino también alarma en la población. En los últimos años en América Latina se han producido cambios en la prevalencia de serogrupos, sien- 
do el más significativo el aumento del serogrupo W135 en la región del Cono Sur (Argentina, Brasil, Uruguay y recientemente Chile). El mejor conocimiento de la epidemiología global a través de las nuevas técnicas de laboratorio moleculares, que permiten la caracterización de genotipos y fenotipos, monitorear la diseminación de cepas de $N$. meningitidis pertenecientes a los linajes hipervirulentos (W135 ST-11/Complejo ET-37) y generar alertas epidemiológicas rápidas son en la actualidad, junto con la disponibilidad de las vacunas conjugadas, las herramientas más relevantes para controlar períodos hiperendémicos o epidémicos de EM.

\section{Resumen}

La enfermedad meningocóccica (EM) producida por Neisseria meningitidis es una causa de alta mortalidad en la niñez. $N$. meningitidis serogrupo W135 (MenW135) es habitualmente asociado en el mundo con el 1 al $8 \%$ de los casos de EM y con una baja tasa de portadores. En 2000 en Arabia Saudita se informó un aumento del MenW135 coincidente con la peregrinación a la Meca, Hajj (cepa Hajj-2000). Mayer y cols. estudiaron cepas MenW135 de brotes relacionados con la peregrinación, y hallaron que todos los casos fueron producidos por el mismo clon hipervirulento (ST-11/complejo ET-37), cepa cuya circulación también se pudo documentar en América Latina. En los últimos años en la región se han producido cambios en la prevalencia de serogrupos, siendo el más significativo el aumento de MenW135 en el Cono Sur. Para la prevención de las infecciones por $N$. meningitidis de los diversos serogrupos incluyendo MenW135, se dispone de la quimioprofilaxis a través del uso de antimicrobianos y de las vacunas cuadrivalentes. El mejor conocimiento de la epidemiología global a través de las nuevas técnicas de laboratorio moleculares, junto con la disponibilidad de las vacunas, son las herramientas más relevantes para controlar períodos hiperendémicos o epidémicos de EM.

\section{Referencias bibliográficas}

1. Rosenstein N, Perkins B, Stephens D, Popovic T, Hughes JM. Meningococcal disease. N England J Med 2001; 344: 1378-88.

2. Caugant D A. Population genetics and molecular epidemiology of Neisseria meningitidis. Apmis 1998; 106: 505-25.

3. Maiden M C J, Bygraves J A, Feil E, Morelli G, Russell J E, et al. Multilocus sequence typing: a portable approach to the identification of clones within populations of pathogenic microorganisms. Proc Natl Acad Sci USA 1998; 95: 3140-5.

4. Yazdankhah S P, Kriz P, Tzanakaki G, Kremastinou J, Kalmusova J, et al. Distribution of serogroups and genotypes among disease-associated and carried isolates of Neisseria meningitidis from the Czech Republic, Greece, and Norway. J Clin Microbiol 2004; 42: 5146-53.

5. Brandstetter R D, Blair R J, Roberts R B. Neisseria meningitidis serogroup W135 disease in adults. JAMA 1981; 246: 2060-1.

6. Mayer L W, Reeves M W, Al-Hamdan N, Sacchi C T, Taha M K, Ajello G W, et al. Outbreak of W135 meningococcal diseases in 2000: Not emergence of a new W135 strain but clonal expansion within the electophoretic type37 complex . J Infect Dis 2002; 185: 1596-605.

7. Popovic T, Sacci C T, Reeves M W, Whitney A M, Mayer L W, Noble C A, et al. Neisseria meningitidis serogroup W135 isolates associated with the ET-37 complex. Emerg Infect Dis 2000; 6: 428-9.

8. Lemos A P, Harrison L, Lenser M, Sacchi C. Phenotypic and molecular characterization of invasive serogroup W135 Neisseria meningitidis strains from 1990 to 2005 in Brazil. J Infect 2010; 60: 209-17.

9. Stephens D. Conquering the meningococcus. FEMS Microbiol Rev 2007; 31: 3-14.

10. Stephens D, Greenwood B, Brandtzaeg P. Epidemic meningitis, meningococcemia and Neisseria meningitidis. Lancet 2007; 369: 2196-210.

11. Pollard A. Global epidemiology of meningococcal disease and vaccine efficacy. Pediatr Infect Dis J 2004; 23: S274-9.

12. Ministerio Salud Pública Republica de Chile. Situación enfermedad meningocóccica por serogrupo W135. http://epi.minsal.cl/epi/html/ bolets/reportes/Meningitis/W135_Web_SE46A. pdf. (accedido noviembre 2012).

13. American Academy of Pediatrics Meningococccal infections. In Pickering LK, Baker CJ, Kimberlin DW, Long SS, eds. Red Book 2012 Report of the Committee on Infectious Diseases. Elk Grove Village, IL. American Academy of Pediatrics; 2012: 500-9.

14. Ibarz-Pavón A B, Lemos A P, Gorga M C, Regueira M, SIREVA II Working Group, Gabastou J. M. Laboratory based surveillance of Neisseria menigitidis isolates from diseases cases in Latin American and Caribbean countries, SIREVA II 2006-2010. PLoS One 2012; 7: e44102.

15. Chiavettal L, Chávez E, Ruzic A, Mollerach M, Requeira M. Vigilancia de Neisseria meningitidis en Argentina 1993-2005: distribución de serogrupos, serotipos y serosubtipos causantes de enfermedad invasiva. Rev Argent Microbiol 2007; 39: 21-7.

16. Efron A M, Sorohuet C, Salcedo C, Abad R, Requeira M, Vázquez J A. W135 Invasive meningococcal strain spreading in South
America: Significant increase in incidence rate in Argentina. J Clin Microbiol 2009; 47: 197980 .

17. Sordelli N, Orlando N, Neyro S, Echave C, Procopio A, Fallo A, et al. Artritis meningococcicas primarias en pediatría. Presentación de nueve casos. Arch Argent Pediatr 2011; 109 (2): 150-9.

18. Safadi M A, Cintra O A. Epidemiology of meningococcal diseases in Latin America: current situation and opportunities for prevention. Neurol Res 2010; 32: 263-71.

19. Baethgen L F, Weidlich L, Moraes C, Klein C, Nunes L S, Cafrune P I, et al. Epidemiology of meningococcal disease in southern Brazil from 1995 to 2003, and molecular characterization of Neisseria meningitidis using multilocus sequence typing. Trop Med Int Health 2008; 13: 31-40.

20. Weidlich L, Baethgen L F, Mayer L W, Moraes C, Klein C C, Nunes L S, et al. High prevalence of Neisseria meningitidis hypervirulent lineages and emergence of W135:P1.5,2:ST-11 clone in Southern Brazil. J Infect 2008; 57: 324-31.

21. Barroso D B, Rebelo M C. Recognition of the epidemiological significance of Neisseria meningitidis capsular serogroup W135 in the Rio de Janeiro region, Brazil. Mem Inst Oswaldo Cruz 2007; 6: 773-5.

22. Gorla M C, Lemos A P, Grando I, de Moraes C, de Oliveira Portela C, Zanelato C, et al. Epidemiology of serogroup W135 meningococcal disease in Brazil. 18 ${ }^{\text {th }}$ International Pathogenic Neisseria Conference (IPNC); September 9-14, 2012; Würzburg, Germany.

23. Informe Regional de SIREVA II, 2011. 
Datos por país y por grupos de edad sobre las características de los aislamientos de Streptococcus pneumoniae, Haemophilus influenzae y Neisseria meningitidis en procesos invasores. Washington, D.C.: OPS, 2012.

24. Gallegos Ulloa D. Unidad de Vigilancia, Departamento de Epidemiología, División de Rectoría y Regulación Sanitaria, Informe Vigía Ministerio Salud Pública CHILE. http://epi. minsal.cl/epi

25. O'Ryan M, Vergara R, Pérez C, Jiménez J, Valenzuela M T para el CAVEI Comunicado Comisión Asesora en Vacunas y Estrategias de Inmunización (CAVEI) en relación al aumento de infecciones por Neisseria meningitidis. Ministerio Salud Pública República de Chile. Octubre 5, 2012 .
26. Mac Lennan J M, Urwin R, Obaro S, Griffiths D, Greenwood B, Maiden M C. Carriage of serogroup W135, ET-37 meningococci in the Gambia: implications for immunization policy?. Lancet 2000; 356 : 1078.

27. Centers for Diseases Control and Prevention. Travelers Health http://wwwnc.cdc.gov/travel/

28. Bilukha O, Rosenstein N, National Center for Infectious Diseases, Centers for Disease Contro and Prevention (CDC). Prevention and Control of Meningococcal Diseases. Recommendations of the Advisory Committee on Immunization Practices (ACIP). MMWR Morb Mortal Wkly Rep Recomm Rep 2005; 54: 1-21.

29. MacNeil J R, Cohn A Z, Zell E R, Schmink S, Miller E, Clark T, et al. Early estimate of the effectiveness of quadrivalent meningococcal conjugate vaccine. Pediatric Infect Dis J 2011; 30: 451-5.

30. CDC Yellow Book 2012 (wwwnc.cdc.gov/ travel/page/yellowbook-2012-home.htm )

31. Meningococcal Vaccines: WHO position paper, November 2011. Wkly Epidemiol Rec 2011; 86: 521-39.

32.- Paganini H, López E L, Monaco A, Praino M L, Sarkis C, Vozza L, et al. Multicenter study of meningococcal disease in children in pediatric hospitals in Argentina. International Journal of Infectious Diseases 2010; Conference: 14th International Congress on Infectious Diseases (ICID) Miami, FL United States. March 2010. Conference Publication: 14:e419-e20. 